\title{
Can Combining Femoral and Acetabular Morphology Parameters Improve the Characterization of Femoroacetabular Impingement?
}

\author{
Heinse W. Bouma MD, Tom Hogervorst MD, PhD, \\ Emmanuel Audenaert MD, PhD, Peter Krekel PhD, \\ Paulien M. van Kampen PhD
}

Published online: 11 November 2014

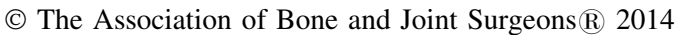

\begin{abstract}
Background Femoroacetabular impingement (FAI) presupposes a dynamic interaction of the proximal femur and acetabulum producing clinical symptoms and chondrolabral damage. Currently, FAI classification is based on alpha angle and center-edge angle measurements in a single plane. However, acetabular and femoral version and neck-shaft angle also influence FAI. Furthermore, each of
\end{abstract}

Each author certifies that he or she, or a member of his or her immediate family, has no funding or commercial associations (eg, consultancies, stock ownership, equity interest, patent/licensing arrangements, etc) that might pose a conflict of interest in connection with the submitted article.

All ICMJE Conflict of Interest Forms for authors and Clinical Orthopaedics and Related Research ${ }^{\circledR}$ editors and board members are on file with the publication and can be viewed on request.

Clinical Orthopaedics and Related Research ${ }^{\mathbb{R}}$ neither advocates nor endorses the use of any treatment, drug, or device. Readers are encouraged to always seek additional information, including FDAapproval status, of any drug or device prior to clinical use. Each author certifies that his or her institution approved or waived approval for the human protocol for this investigation and that all investigations were conducted in conformity with ethical principles of research.

This work was performed at Haga Hospital, the Hague, The Netherlands.

H. W. Bouma ( $\square)$, T. Hogervorst, P. M. van Kampen Department of Orthopaedic Surgery, Haga Hospital, Sportlaan 600, 2566 MJ The Hague, The Netherlands

e-mail: heinsebouma@gmail.com

E. Audenaert

Department of Orthopaedic Surgery, Ghent University Hospital, Ghent, Belgium

P. Krekel

Clinical Graphics, Delft, The Netherlands these parameters has a reciprocal interaction with the others; for example, a shallow acetabulum delays impingement of the femoral head with the acetabular rim. Questions/purposes We introduce the new parameter "omega zone," which combines five parameters into one: the alpha and center-edge angles, acetabular and femoral version, and neck-shaft angle. We sought to determine whether the omega zone could differentiate patients with FAI from (1) normal control subjects (alpha $<55^{\circ}$ ), but also from (2) control subjects with elevated alpha angles $\left(\geq 55^{\circ}\right)$

Methods We evaluated CT data of 20 hips of male patients with symptomatic cam-type FAI and of 35 male hips extracted from 110 anonymized CT scans for vascular diagnosis. We excluded hips with osteoarthritis, developmental dysplasia, or coxa profunda (center-edge angle $20^{\circ}-$ $45^{\circ}$ on AP pelvic view or corresponding coronal CT views). With dedicated software, femoral and pelvic orientation was standardized; we tested the omega zone in four hip positions in three distinct groups: patients with cam-type FAI (alpha $>60^{\circ}$ ) and control subjects with normal $\left(<55^{\circ}\right)$ and high alpha angles $\left(\geq 55^{\circ}\right)$.

Results The omega zone was smaller in patients with cam-type FAI than normal control subjects (alpha angle $<55^{\circ}$ ) at $60^{\circ}$ and $90^{\circ}$ of flexion (mean, $12 \%$; $95 \%$ confidence interval $[\mathrm{CI}], 7-17 ; \mathrm{p}=0.008 ;$ Cohen's $\mathrm{d}=9 \% ; 95 \% \mathrm{CI}, 4-13 ; \mathrm{p}=0.003)$. Furthermore, the omega zone was smaller in all positions in patients with cam-type FAI than control subjects with high alpha angles $\left(0^{\circ} \mathrm{p}=0.017,30^{\circ} \mathrm{p}=0.004, \quad 60^{\circ} \mathrm{p}=0.004, \quad 90^{\circ}\right.$ $\mathrm{p}=0.007$ ). In contrast, the omega zone did not differ between control subjects with normal or high alpha angles. In all hips, the omega zone decreased with flexion, corresponding to a decrease in remaining impingement-free motion with flexion. 
Conclusions The omega zone visualizes and quantifies the interaction of the proximal femur and acetabulum. The omega zone differed between patients with cam-type FAI and control subjects with high alpha angles $\left(\geq 55^{\circ}\right)$, who could not be distinguished based on alpha angle alone. For hip-preserving surgery, it can help surgeons decide whether to address the femur, the acetabulum, or both.

Level of Evidence Level III, diagnostic study.

\section{Introduction}

Hip osteoarthritis (OA) may result from dynamic interaction of the proximal femur and acetabulum through the mechanism of femoroacetabular impingement (FAI) [8]. An example is the movement of an aspherical femoral head into a (more) spherical acetabulum causing damage to the cartilage at the chondrolabral junction. In this concept, the morphology of the femoral head and the acetabulum has a reciprocal interaction: a shallow acetabulum (dysplasia) decreases or delays impingement of the femoral head or head-neck junction with the acetabular rim, and, conversely, a more spherical femoral head will decrease or delay impingement in a deep acetabulum (coxa profunda) [3].

Conceptually, current quantification and classification of FAI are based on femoral concavity measurements (like the alpha angle) for the cam morphotype and acetabular coverage measurements (like center-edge [CE] angle) for the pincer morphotype. However, such classification of FAI morphotypes does not reflect the dynamic and reciprocal interaction of the joint components, where proximal femoral morphology can compensate for acetabular morphology and vice versa. Furthermore, morphological parameters that determine how the proximal femur and acetabulum are positioned relative to each other also play a role in FAI, as does their interaction. These parameters are acetabular and femoral version and femoral neck shaft angle $[4,15]$. For example, a femur with low neck-shaft angle (varus) impinges earlier on the acetabular rim with flexion-internal rotation than a femur with higher neckshaft angle (closer to $135^{\circ}$ ). Ideally then, diagnosis and classification of FAI should address all five parameters, namely, alpha and CE angles, femoral and acetabular version, and femoral neck-shaft angle and, given the dynamic nature of FAI, do so in three dimensions.

We therefore developed a parameter that visualizes and quantifies the joint femoral and acetabular morphology and their interaction: the omega zone. This new parameter combines five parameters in multiple planes into a single new parameter and reflects their interaction. We tested the omega zone in three groups: patients with symptomatic cam-type FAI, control subjects with high $\left(\geq 55^{\circ}\right)$ alpha
Table 1. Characteristics of the three subject groups

\begin{tabular}{llll}
\hline Group & FAI & C-high $\geq 55^{\circ}$ & C-low $<55^{\circ}$ \\
\hline $\begin{array}{c}\text { Number of } \\
\text { subjects }\end{array}$ & 13 & 16 & 7 \\
$\begin{array}{c}\text { Number of } \\
\text { hips }\end{array}$ & 20 & 26 & 9 \\
$\begin{array}{c}\text { Mean age } \\
\text { (years) }\end{array}$ & $\begin{array}{c}\text { 26-36) } \\
\text { (95\% CI, }\end{array}$ & $\begin{array}{c}50 *(95 \% \mathrm{CI}, \\
41-58)\end{array}$ & $\begin{array}{c}61^{*}(95 \% \mathrm{CI}, \\
50-71)\end{array}$ \\
\hline
\end{tabular}

* Mean age C-high $\mathrm{p}=0.002$, mean age $\mathrm{C}$-low $\mathrm{p}=0.000$ with analysis of variance and Bonferroni post hoc analysis compared with FAI; FAI = femoroacetabular impingement; $\mathrm{C}$-high $=$ subjects with alpha angle $\geq 55^{\circ}$; C-low $=$ subjects with alpha angle $<55^{\circ}$.

angles, and control subjects with normal $\left(<55^{\circ}\right)$ alpha angles. Specifically, we sought to determine whether the omega zone could differentiate (1) between patients with cam-type FAI and normal control subjects (alpha $<55^{\circ}$ ); and (2) between patients with cam-type FAI and control subjects with elevated alpha angles $\left(\geq 55^{\circ}\right)$.

\section{Patients and Methods}

All data were retrospectively acquired from anonymized databases. The institutional review boards of the involved hospitals decided no ethical approval was necessary. Hips with radiographic joint space narrowing (Tönnis Grade $\geq 2$ [21]), posttraumatic abnormalities, or any osteophyte formation that could influence measurements were excluded.

\section{Patients With Femoroacetabular Impingement}

The CT data of 13 male patients with symptomatic camtype FAI (20 hips; Table 1) were included. The diagnosis of cam-type FAI was made by three experienced clinicians (EA, RMB, TH) based on patient history, a positive impingement test on clinical examination [13], and/or radiographic signs of cam impingement (alpha $\geq 60^{\circ}$ in 11 , alpha $>55^{\circ}$ in two patients), and/or arthroscopy. The included patients were derived from a total of 31 patients with FAI who were analyzed with CT scans in the clinician's practice over a 3-month period. From this group of CT scans we excluded patients with mixed-type FAI (maximum $\mathrm{CE}$ angle $>45^{\circ}$ and aspherical femoral head with alpha angle $\geq 55^{\circ}$ ) or coxa profunda (maximum CE angle $>45^{\circ}$ and spherical femoral head with alpha angle $<55^{\circ}$ ) or degenerative changes Tönnis Grade 2 or higher and/or incomplete CT scans, for example when femoral condyles were not included in the scans. All data were anonymized. 
Control Subjects (C-high, C-low)

Anonymized CT data from databases for vascular screening of two different hospitals were divided into two groups based on alpha angle. Twenty-three male subjects were randomly selected from a total of 110 male and female subjects. Exclusion criteria were identical to those of the FAI patient group with the addition of exclusion of $\mathrm{CE}$ angle below $20^{\circ}$ or over $45^{\circ}$ on AP pelvic radiographs or corresponding coronal CT views. Thirty-five hips were included. Using the most commonly used cutoff value of $55^{\circ}$ for the alpha angle at any position among the $12,1,2$, and 3 o'clock positions, we divided the control group into subjects with alpha angle $\geq 55^{\circ}$ (C-high, 26 hips) and subjects with alpha angle $<55^{\circ}$ (C-low, 9 hips; Table 1$)$.

\section{Methods/Software}

\section{CT Imaging}

All scans for FAI were made between 2010 and 2013; all scans for the control group were made between 2005 and 2012. CT scans were performed on three different CT scanners (Siemens Healthcare, Erlangen, Germany; Toshiba Medical Systems Corporation, Tochigi-ken, Japan; GE Healthcare; Little Chalfont, Buckinghamshire, UK) with slice thickness varying between 0.625 and $2.5 \mathrm{~mm}$ and a gantry tilt of $0^{\circ}$. For the hip (acetabular roof to head-neck junction), maximal slice thickness was $2 \mathrm{~mm}$. All scans contained the pelvic bone, proximal femur, and slices of the knee containing the posterior condyles.

\section{Three-dimensional Model}

The hip was semiautomatically segmented using dedicated FAI software (Articulis ${ }^{\mathrm{TM}}$; Clinical Graphics, Delft, The Netherlands) that has been previously validated for reliability and accuracy [19]. The software performed the three-dimensional (3-D) segmentation of the CT scans. Only the radiodense osseous structures were digitally subtracted, rendering a 3-D image of a hemipelvis containing the left or right hip. All left hips were mirrored to represent a right hip. A clinical expert checked whether each segmentation contained all osseous contours of the CT scan. Thereafter, Articulis ${ }^{\mathrm{TM}}$ linked the bones as a joint; the $\mathrm{x}-, \mathrm{y}-$, and $\mathrm{z}$-axes were determined according to the recommendations of the International Society of Biomechanics (ISB) [22]. An exception was made for the $\mathrm{x}$-axis of the pelvis (ie, the horizontal or transversal plane), which we defined as the plane from the posteroinferior iliac spine to the anterosuperior spine, corresponding better to the in vivo (standing) $\mathrm{x}$-axis of the human pelvis. A pelvic plane is established and the pelvis (and pelvic tilt) was standardized according to the recommendations of the ISB in all scans.

\section{Alpha and Center-edge Angle, Femoral Version,} Acetabular Version, and Inclination Measurement

Clockwise alpha angles were measured at seven positions at $30^{\circ}$ intervals in the upper hemisphere of the femoral head as described by Nötzli et al. [17]. Briefly, alpha angles were measured between two lines originating in the center of the regression sphere of the femoral head. One line is drawn from this center along the femoral neck axis, the other from this center to the point where the osseous contour of the femoral head exits the regression sphere, and this is done at each of the seven positions at $30^{\circ}$ intervals. Because of the location of the cam on the anterosuperior head-neck junction, only the four positons from 12 to 3 o'clock positions were used.

The CE angle was measured between the plane of the $y$-axis of the pelvis and a line from the center of a regression sphere in the acetabulum to the osseous rim of the acetabulum. This measurement was performed in $30^{\circ}$ steps in the upper hemisphere of the acetabulum from $0^{\circ}$ to $180^{\circ}$. Thus, we measured the CE angle on seven locations in steps of $30^{\circ}$ from anterior to posterior. This measurement is negative for the $0^{\circ}$ position as a result of the anteversion of the acetabulum (Fig. 1). Analogous to the alpha angle, only the four positions from $30^{\circ}$ to $120^{\circ}$ were used, because this is the location where cam impingement usually occurs. The acetabular version and inclination are derived from the coordinate frame by the software comparing it with the pelvic plane. The femoral version was measured by adding two measurement points on the posterior condyles, one medial and one lateral. The software automatically determines the femoral neck version by comparing the coordinate frame in relation to these points. When comparing isolated parameters between the groups, clear differences are seen for the alpha angle at 1,2 , and 3 o'clock as a result of the definition of the groups (Table 2). Comparing the two control groups, smaller differences are seen for femoral neck-shaft angle, CE angles, and femoral version. Although these parameters do not differ substantially when examined in isolation, their combined effects may explain the difference between individual hips whether FAI occurs or not. For example, the higher alpha angles of the C-high group may be compensated by small increases in neck-shaft angle and acetabular version and a small decrease in CE angles (Table 2). 

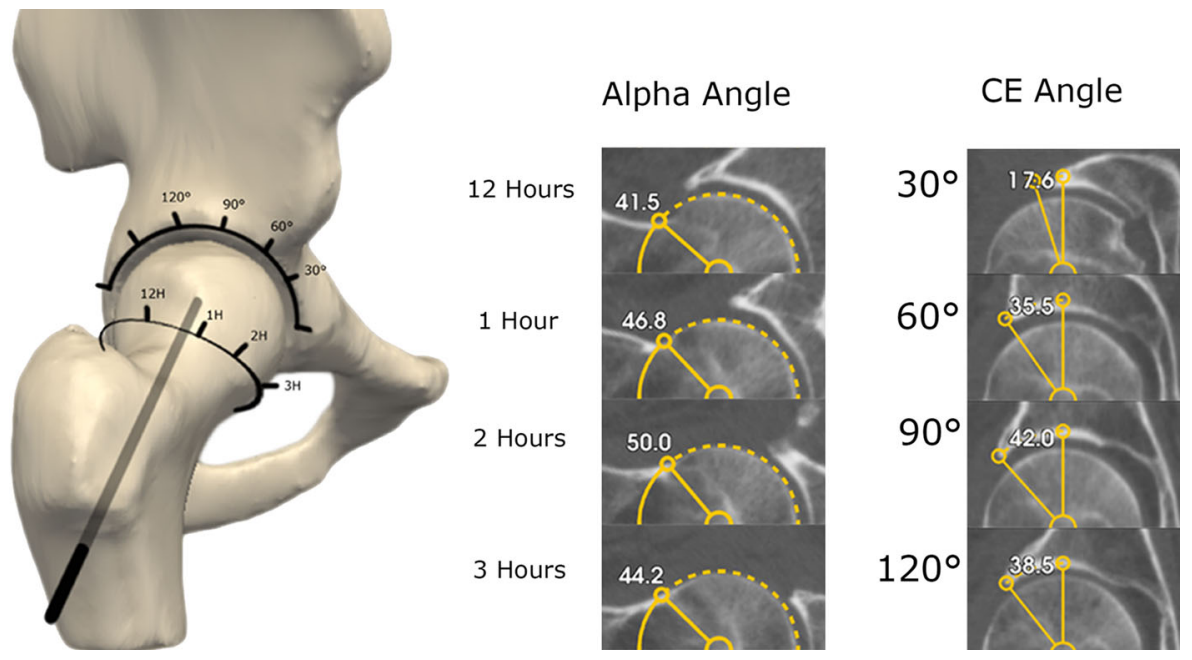

Fig. 1 Alpha angle and Center-edge angle measurements are shown in steps of $30^{\circ}$ in relation to the anatomical coordinate system according to the International Society of Biomechanics.

Table 2. All measured parameters

\begin{tabular}{|c|c|c|c|c|c|c|c|}
\hline Parameter & Position & FAI & $\begin{array}{l}\text { Alpha } \\
\text { angle } \geq 55^{\circ}\end{array}$ & $\begin{array}{l}\mathrm{p} \text { value } \\
\text { versus FAI }\end{array}$ & $\begin{array}{l}\text { Alpha } \\
\text { angle }<55^{\circ}\end{array}$ & $\begin{array}{l}\mathrm{p} \text { value } \\
\text { versus FAI }\end{array}$ & $\begin{array}{l}\mathrm{p} \text { value alpha angle } \geq 55^{\circ} \\
\text { versus alpha angle }<55^{\circ}\end{array}$ \\
\hline \multirow[t]{4}{*}{ Alpha angles } & 12 hours & $50(45-55)$ & $48(47-49)$ & 1.000 & $45(41-48)$ & 0.187 & 0.636 \\
\hline & 1 hours & $61(57-65)$ & $60(58-62)$ & 1.000 & $50(47-51)$ & 0.000 & 0.000 \\
\hline & 2 hours & $63(60-66)$ & $55(53-57)$ & 0.000 & $49(46-50)$ & 0.000 & 0.010 \\
\hline & 3 hours & $53.3(51-56)$ & $47.5(45-49)$ & 0.000 & $43.3(40-46)$ & 0.000 & 0.177 \\
\hline \multicolumn{2}{|c|}{ Femoral version } & $11(7-15)$ & $8(6-10)$ & 0.488 & $9(4-14)$ & 1.000 & 1.000 \\
\hline \multicolumn{2}{|c|}{ Neck-shaft angle } & $131(129-133)$ & $134(132-136)$ & 0.088 & $131(128-134)$ & 1.000 & 0.23 \\
\hline \multirow[t]{4}{*}{$\mathrm{CE}$ angle } & $30^{\circ}$ & $8(2-13)$ & $6(1-11)$ & 1.000 & $19(14-24)$ & 0.052 & 0.014 \\
\hline & $60^{\circ}$ & $30(26-34)$ & $29(26-32)$ & 1.000 & $34(27-40)$ & 0.909 & 0.522 \\
\hline & $90^{\circ}$ & $38(35-41)$ & $35(32-38)$ & 0.342 & $39(35-43)$ & 1.000 & 0.434 \\
\hline & $120^{\circ}$ & $34(31-38)$ & $29(26-33)$ & 0.140 & $34(27-40)$ & 1.000 & 0.512 \\
\hline \multicolumn{2}{|l|}{ Inclination } & $42(40-45)$ & $41(39-44)$ & 1.000 & $42(38-47)$ & 1.000 & 1.000 \\
\hline \multicolumn{2}{|c|}{ Acetabular version } & $13(11-16)$ & $14(12-16)$ & 1.000 & $12(8-15)$ & 1.000 & 0.937 \\
\hline
\end{tabular}

Means with 95\% confidence intervals in parentheses. Probability values given are analysis of variance with Bonferroni post hoc analysis with significance at $\mathrm{p} \leq 0.05 ; \mathrm{FAI}=$ femoroacetabular impingement; $\mathrm{CE}=$ center-edge.

\section{Omega Zone}

The omega zone represents the impingement-free ROM of the head in the acetabulum and is defined as the area between the femoral head coverage area and alpha area. Thus, by definition, the omega zone is influenced not only by alpha and CE angles, but also by the position of the femoral head in the acetabulum. Femoral version and femoral neck-shaft angle are both incorporated in the alpha zone because both influence the position of the femoral head in the acetabulum. Similarly, acetabular version is incorporated in the femoral head coverage area. These measurements are not calculated explicitly, but follow from the coordinate frames that we choose for calculating the omega zone.
We used a representation of the femoral head as a circle seen from cranially, as introduced by Dandachli et al. [5]. In this circle we projected three areas as percentages of this circle. One area represents acetabular coverage (coverage area), a second represents the aspherical area of the femoral head (alpha area), and the third area, named the "omega zone," is the area between the coverage and alpha areas.

Analogous to the acetabulum, the center of the femoral head and its radius were determined with a regression sphere fitted on 400 random points placed on the proximal half of the femoral head. The fovea was explicitly excluded from this point set. The cam lesion is defined as bony formation outside this sphere, ie, the asphericity of the femoral head. The software quantifies the cam lesion as a percentage of the femoral head surface, the alpha area. 


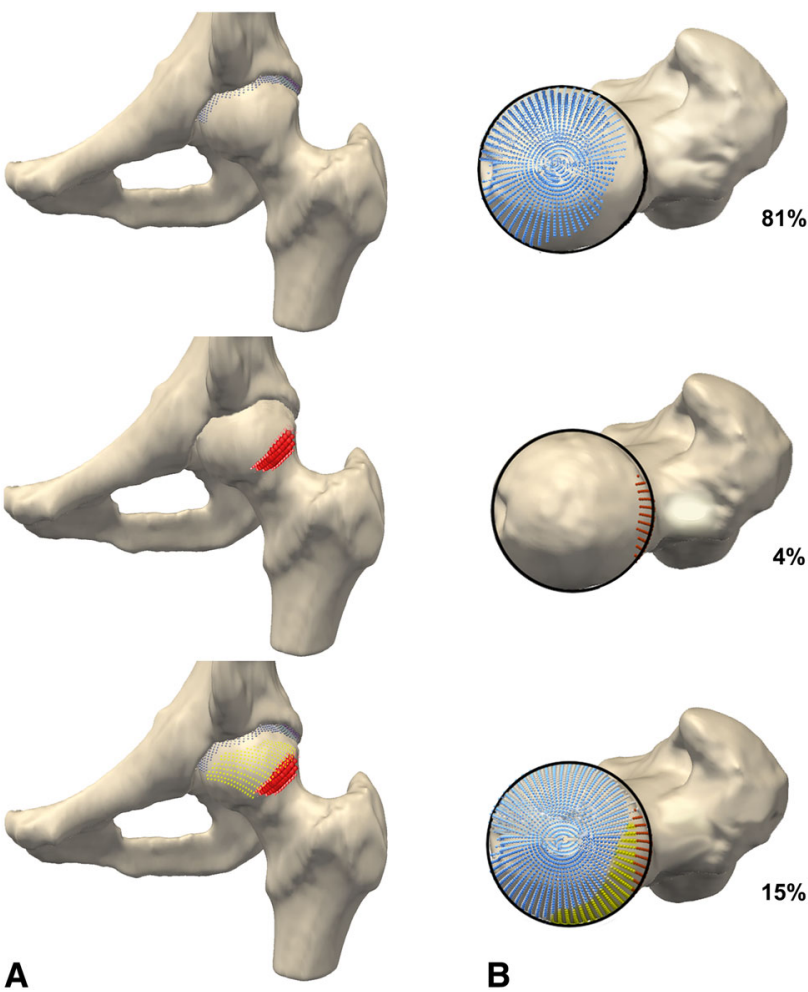

Fig. 2A-B Coverage area (blue), alpha area (red), and omega zone (yellow) in AP (A) and craniocaudal (B) view with the hip in $0^{\circ}$ flexion is shown.

Acetabular coverage is calculated based on Dandachli et al. [5]. The acetabular coverage is also a percentage of the femoral head surface (Fig. 2).

With the femoral head represented as a circle as seen cranially, we projected the acetabular coverage and the alpha area as percentages of this circle and thus a twodimensional representation of a 3-D shape. The area remaining between the coverage and alpha areas is named the omega zone (Fig. 2).

Furthermore, flexion of the hip is simulated and corresponding percentages of the alpha area and omega zone are calculated. By putting the femur in flexion, the alpha zone of the anterior hemisphere of the femoral head is calculated. As such, both a superior and anterior cam would be detected, increasing the alpha area and decreasing the omega zone. Further rotations are not necessary as the alpha area enters the omega zone in $90^{\circ}$ of flexion. We calculated the omega zones for neutral, $30^{\circ}, 60^{\circ}$, and $90^{\circ}$ of hip flexion (Fig. 3) and compared them among the three groups (FAI, C-high, and C-low). The motion simulation was based on methods according to ISB recommendations with steps of $1^{\circ}$ of flexion with collision detection between the acetabulum and femoral head. Translation of the femoral head in motion simulation was accounted for with the equidistant method [18], but this does not affect the alpha area or omega zone.

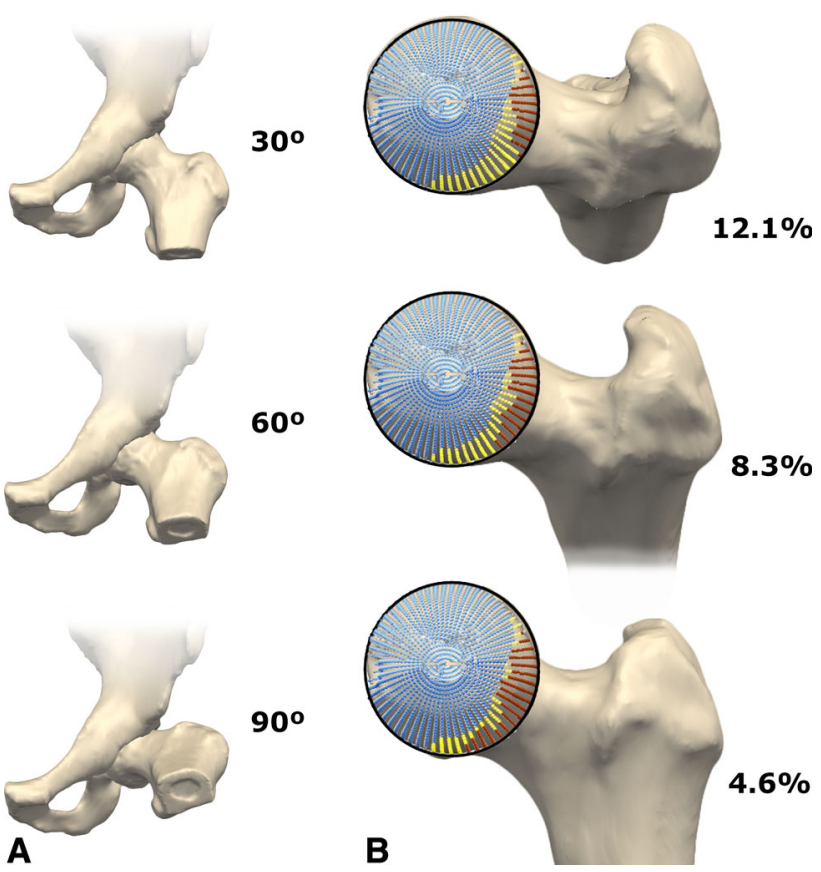

Fig. 3A-B Coverage area (blue), alpha area (red), and omega zone (yellow) in AP (A) and craniocaudal (B) view with the hip in $30^{\circ}, 60^{\circ}$, and $90^{\circ}$ of flexion is shown. With increasing flexion, the circle projected over the femoral head shifts to the anterior femoral head (bottom right).

\section{Statistics and Calculations}

Statistical analysis was performed using SPSS 17.0 (SPSS Inc, Chicago, IL, USA). The Shapiro-Wilk test showed normal distribution for the various measured parameters of the hip, except for the omega zone in the C-high group. To establish differences in alpha angles, CE angles, anteversion and inclination, and femoral version, we used one-way analysis of variance with a post hoc Bonferroni correction test. A $p$ value of $<0.05$ was considered significant. For the omega zone, we used the Kruskal-Wallis $\mathrm{H}$ test with a post hoc Mann-Whitney $U$ test for effect size. Also we conducted a Bonferroni correction; in this case a significance level of $p<0.017$ was used for comparison of the three groups. For the calculation of the intraclass correlation coefficient (ICC), $10 \mathrm{CT}$ scans were segmented with Articulis $^{\mathrm{TM}}$ and an untrained medical student drew the axes. Measurements of the various parameters showed an excellent interobserver agreement with an ICC of $\geq 0.9$.

\section{Results}

The Kruskal-Wallis test for the omega zone showed a difference for all groups at $0^{\circ}, 30^{\circ}, 60^{\circ}$, and $90^{\circ}$ of flexion with $\mathrm{p}$ values of $0.038,0.008,0.003$, and 0.002 , 
Table 3. Omega zone in percentage of femoral head circle

\begin{tabular}{|c|c|c|c|c|c|c|c|c|c|c|}
\hline \multirow{2}{*}{$\begin{array}{l}\text { Omega zone } \\
\text { Flexion }\end{array}$} & \multicolumn{2}{|l|}{ FAI } & \multicolumn{4}{|c|}{ C-high $\geq 55^{\circ}$} & \multicolumn{4}{|c|}{ C-low $<55^{\circ}$} \\
\hline & Mean & $95 \% \mathrm{CI}$ & Mean & $95 \% \mathrm{CI}$ & $\mathrm{p}$ value* & $\mathrm{d}$ & Mean & $95 \% \mathrm{CI}$ & $\mathrm{p}$ value* & $\mathrm{d}$ \\
\hline $0^{\circ}$ & 18 & $15-22$ & 23 & $19-27$ & 0.017 & -0.55 & 23 & $20-27$ & 0.069 & -0.86 \\
\hline $30^{\circ}$ & 16 & $11-20$ & 22 & $19-26$ & 0.004 & -0.82 & 23 & $20-27$ & 0.027 & -1.11 \\
\hline $60^{\circ}$ & 12 & $7-17$ & 20 & $18-22$ & 0.004 & -0.97 & 23 & $19-26$ & 0.008 & -1.31 \\
\hline $90^{\circ}$ & 9 & $4-13$ & 16 & $13-19$ & 0.007 & -0.87 & 19 & $17-22$ & 0.003 & -1.50 \\
\hline
\end{tabular}

* Compared with FAI; FAI = femoroacetabular impingement; C-high $=$ subjects with alpha angle $\geq 55^{\circ}$; C-low $=$ subjects with alpha angle $<55^{\circ}$; for the post hoc Mann-Whitney U test, significance level was set at $\mathrm{p}<0.017$ with Bonferroni correction; $\mathrm{d}=\mathrm{Cohen}$ 's $\mathrm{d}$ effect size.

respectively. Patients with cam-type FAI were found to have a mean omega zone of $12 \%$ (95\% confidence interval [CI], 7-17) and $9 \%(95 \% \mathrm{CI}, 4-13)$ at $60^{\circ}$ and $90^{\circ}$ of flexion, respectively. At these degrees of flexion, control subjects with normal $\left(<55^{\circ}\right)$ alpha angles showed a larger mean omega zone of $23 \%(95 \% \mathrm{CI}, 19-26 ; \mathrm{p}=0.008)$ and $19 \%$ (95\% CI, 17-22; $\mathrm{p}=0.003$ ) (Table 3).

The control subjects with elevated $\left(\geq 55^{\circ}\right)$ alpha angles also showed a larger mean omega zone of $20 \%(95 \% \mathrm{CI}$, $18-22 ; \mathrm{p}=0.004)$ and $16 \%(95 \% \mathrm{CI}, 13-19 ; \mathrm{p}=0.007)$ for $60^{\circ}$ and $90^{\circ}$ of flexion, respectively, compared with the patients with FAI. Furthermore, the mean omega zone at $0^{\circ}$ and $30^{\circ}$ was larger with $23 \%$ (95\% CI, 19-27; p = 0.017) and $22 \%(95 \%$ CI, 19-26; $\mathrm{p}=0.004)$, respectively, whereas the mean omega zone in patients with FAI was $18 \%$ (95\% CI, 15-22) and 16\% (95\% CI, 11-20) (Table 3). In contrast, the omega zone was similar in both control groups at any of the flexion positions $\left(0^{\circ} \mathrm{p}=0.806,30^{\circ}\right.$ $\left.\mathrm{p}=0.925,60^{\circ} \mathrm{p}=0.345,90^{\circ} \mathrm{p}=0.136\right)$.

\section{Discussion}

We introduce the omega zone to visualize and quantify the contribution of both sides of the hip to FAI. The omega zone combines alpha and CE angles, but also femoral and acetabular version and femoral neck-shaft angle, giving a more comprehensive, quantitative parameter for hip morphology and FAI. The omega zone is CT-based and can be determined in any position of the hip, reflecting the dynamic interaction between the proximal femur and acetabulum.

The current study is intended as proof of principle. Further work is needed on larger patient groups with more varied hip morphologies. Nevertheless, we acknowledge several limitations in our study. First, the control group consisted of a small number of subjects with an alpha angle of $55^{\circ}$ or smaller. In a CT-based study we could not easily perform scans on males with low alpha angles to enlarge this group. Nevertheless, significant differences and large effect sizes were still found compared with the FAI group. Women with low alpha angles were excluded to reduce confounders in our comparison with the all-male group of patients with cam-type FAI. Other impingement patterns such as pincer impingement or mixed-type impingement were not assessed in this first study for the same reason, but we expect to find a similar decrease of the omega zone in other impingement patterns. Pincer impingement for example would have larger coverage of the femoral head and therefore a smaller omega zone.

Further, although we distinguished between patients with symptomatic FAI and symptom-free control subjects, we could not obtain the history and clinical examination in the control subjects. Therefore, we cannot be completely certain whether the control subjects had signs or symptoms of the hip, although we assume that reaching their mean age of 53 years without signs of OA offers support they were likely symptom-free in the hip.

Additionally, although MRI-based image analysis for the omega zone is being developed, current data are CTbased, ignoring cartilage and soft tissues. Wyss et al. showed, however, that it is the osseous structures that cause chondrolabral damage in impingement [23]. Although the omega zone addresses all hip morphology parameters relevant for FAI, postural factors such as pelvic tilt and lumbosacral lordosis also influence femoral head position in the acetabulum. Nevertheless, for persons positioned supine in a CT scanner, there is a fairly robust correlation with their standing posture [6, 7]. Finally, the analysis of the omega zone was performed with steps of $30^{\circ}$ of flexion. Maximum flexion or combining internal rotation and subsequent collision of the femoral head or neck with the acetabular rim can be analyzed with further motion simulation but was beyond the scope of the current study that focused on better characterization of morphology, leading to development of the omega zone.

Comparing our findings with others that studied 3-D hip morphology and motion in FAI, we find confirmation on the differences between normal and FAI hips but no integrative parameter such as the omega zone. For example, 
Kubiak-Langer et al. focused on defining the ROM and zone of (acetabular) impingement [14] and described the decreasing range of internal rotation with flexion. Wyss et al. depicted the femoroacetabular interaction as the angle between alpha and CE angle in the flexed hip in an open MRI scanner [23]. This is analogous to the omega zone but in a single plane. Furthermore, hip motion in FAI was studied with virtual hip models by Arbabi et al. [2]. In that study, all morphology parameters were kept constant; only alpha and CE angle were varied to simulate dysplasia, cam-, pincer-, and combined-type morphology. Collision detection algorithms demonstrated two types of penetration of the two rotating bodies (the hip components) with hip motion, named radial and curvilinear, for cam- and pincertype morphology, respectively [2]. Perhaps, for the clinician, the omega zone has the advantage of being more visual.

FAI has been discussed intensively since the initial description of the condition by Ganz and coworkers [9]. Prominent among these discussions is the question whether FAI morphology predictably leads to OA. We think these discussions have lacked the proper measurements to allow comparison between different patient populations and hip morphologies, focusing often on a single parameter, for example the alpha angle. This, however, cannot reflect the dynamic and reciprocal interaction of the joint components.

Correspondingly, although the "overall" association between hip morphology and OA seems well substantiated $[8,11,16]$, the correlation of isolated femoral or acetabular parameters and OA development is clear only for more extreme values and is in fact weak for values used as cutoffs to distinguish between "normal" and "abnormal" morphology $[1,10,16,20]$. For example, the association between OA development and cam morphotype using a cutoff value of $60^{\circ}$ for the lateral alpha angle was found robust only for angles above $83^{\circ}\left(>83^{\circ}\right.$ : end-stage OA in 14 of 56 cases; $60^{\circ}-83^{\circ}$ end-stage $\mathrm{OA}$ in three of 100 cases [1]). Similarly, for developmental dysplasia, only pronounced dysplasia is associated with OA $[12,20]$. We believe "combined" parameters such as the omega zone are better suited for diagnosis and classification of hip disorders than "single" parameters. Nevertheless, we realize morphology alone can never explain the full spectrum of hip symptoms or signs nor whether OA will inevitably develop. Mechanical loading and cartilage characteristics play an important role, including the joint's loading history (frequency and magnitude of all loads across the joint) and ability of cartilage to withstand mechanical stress $[8,11,16]$.

In this study, patients with cam-type FAI were found to have a smaller omega zone than control subjects regardless of whether these control subjects had normal or high alpha angles. In contrast, the omega zone did not differ between control subjects with normal or high alpha angles. These findings can be explained by the integrative nature of the omega zone where one of the five parameters may compensate for another. In all hips, the omega zone decreased with flexion, corresponding to a decrease in remaining impingement-free motion with flexion. The omega zone and its visualization in different positions can help surgeons decide whether to address the femur, acetabulum, or both in treating femoral acetabular impingement.

Acknowledgments We thank Dr R. M. Bloem, Department of Orthopaedics, Reinier de Graaf Gasthuis, Delft, The Netherlands, and Professor R. G. G. H. Nelissen, Department of Orthopaedics of the LUMC, Leiden, The Netherlands, for permission to include patients with FAI and control subjects. We also thank Erik Boekestein for his measurements for the reliability of the omega zone.

\section{References}

1. Agricola R, Reijman M, Bierma-Zeinstra SM, Verhaar JA, Weinans $\mathrm{H}$, Waarsing JH. Total hip replacement but not clinical osteoarthritis can be predicted by the shape of the hip: a prospective cohort study (CHECK). Osteoarthritis Cartilage. 2013;21:559-564.

2. Arbabi E, Chegini S, Boulic R, Tannast M, Ferguson SJ, Thalmann D. Penetration depth method-novel real-time strategy for evaluating femoroacetabular impingement. J Orthop Res. 2010;28:880-886.

3. Audenaert EA, Peeters I, Vigneron L, Baelde N, Pattyn C. Hip morphological characteristics and range of internal rotation in femoroacetabular impingement. Am J Sports Med. 2012;40:1329-1336.

4. Bardakos NV, Villar RN. Predictors of progression of osteoarthritis in femoroacetabular impingement: a radiological study with a minimum of ten years follow-up. J Bone Joint Surg Br. 2009;91:162-169.

5. Dandachli W, Kannan V, Richards R, Shah Z, Hall-Craggs M, Witt J. Analysis of cover of the femoral head in normal and dysplastic hips: new CT-based technique. J Bone Joint Surg Br. 2008;90:1428-1434

6. DiGioia AM, Hafez MA, Jaramaz B, Levison TJ, Moody JE. Functional pelvic orientation measured from lateral standing and sitting radiographs. Clin Orthop Relat Res. 2006;453:272-276.

7. Eilander W, Harris S, Henkus H, Cobb J, Hogervorst T. Functional acetabular component position with supine total hip replacement. Bone Joint J. 2013;95:1326-1331.

8. Ganz R, Leunig M, Leunig-Ganz K, Harris WH. The etiology of osteoarthritis of the hip: an integrated mechanical concept. Clin Orthop Relat Res. 2008;466:264-272.

9. Ganz R, Parvizi J, Beck M, Leunig M, Notzli H, Siebenrock KA. Femoroacetabular impingement: a cause for osteoarthritis of the hip. Clin Orthop Relat Res. 2003;417:112-120.

10. Hartofilakidis G, Bardakos NV, Babis GC, Georgiades G. An examination of the association between different morphotypes of femoroacetabular impingement in asymptomatic subjects and the development of osteoarthritis of the hip. J Bone Joint Surg.Br. 2011;93:580-586.

11. Hogervorst T, Eilander W, Fikkers JT, Meulenbelt I. Hip ontogenesis: how evolution, genes, and load history shape hip morphotype and cartilotype. Clin Orthop Relat Res. 2012;470: 3284-3296. 
12. Jacobsen S, Sonne-Holm S, Soballe K, Gebuhr P, Lund B. Hip dysplasia and osteoarthrosis: a survey of 4151 subjects from the Osteoarthrosis Substudy of the Copenhagen City Heart Study. Acta Orthop. 2005;76:149-158.

13. Klaue K, Durnin CW, Ganz R. The acetabular rim syndrome. A clinical presentation of dysplasia of the hip. J Bone Joint Surg Br. 1991;73:423-429.

14. Kubiak-Langer M, Tannast M, Murphy SB, Siebenrock KA, Langlotz F. Range of motion in anterior femoroacetabular impingement. Clin Orthop Relat Res. 2007;458:117-124.

15. Ng KG, Lamontagne M, Adamczyk AP, Rahkra KS, Beaulé PE. Patient-specific anatomical and functional parameters provide new insights into the pathomechanism of cam FAI. Clin Orthop Relat Res. $2014 \mathrm{Jul} 22$ [Epub ahead of print].

16. Nicholls AS, Kiran A, Pollard TC, Hart DJ, Arden CP, Spector T, Gill HS, Murray DW, Carr AJ, Arden NK. The association between hip morphology parameters and nineteen-year risk of end-stage osteoarthritis of the hip: a nested case-control study. Arthritis Rheum. 2011;63:3392-3400.

17. Nötzli HP, Wyss TF, Stoecklin CH, Schmid MR, Treiber K, Hodler J. The contour of the femoral head-neck junction as a predictor for the risk of anterior impingement. J Bone Joint Surg Br. 2002;84:556-560.
18. Puls M, Ecker TM, Tannast M, Steppacher SD, Siebenrock KA, Kowal JH. The Equidistant Method-a novel hip joint simulation algorithm for detection of femoroacetabular impingement. Computer Aided Surgery. 2010;15:75-82.

19. Röling M, Visser M, Bloem R, Oei E, Kleinrensink GJ, Pilot P. Non-invasive dynamic identification of femoroacetabular impingement: a cadaveric validation study. Arthroscopy. 2013;29:e193-e194.

20. Terjesen T. Residual hip dysplasia as a risk factor for osteoarthritis in 45 years follow-up of late-detected hip dislocation. $J$ Child Orthop. 2011;5:425-431.

21. Tönnis D. Congenital Dysplasia and Dislocation of the Hip in Children and Adults. Vol 101. Berlin, Germany: Springer; 1984.

22. Wu G, Siegler S, Allard P, Kirtley C, Leardini A, Rosenbaum D, Whittle M, D'Lima DD, Cristofolini L, Witte H, Schmid O, Stokes I. ISB recommendation on definitions of joint coordinate system of various joints for the reporting of human joint motion-part I: ankle, hip, and spine. International Society of Biomechanics. J Biomech. 2002;35:543-548.

23. Wyss TF, Clark JM, Weishaupt D, Notzli HP. Correlation between internal rotation and bony anatomy in the hip. Clin Orthop Relat Res. 2007;460:152-158. 